\title{
Acute Myocarditis in a Patient Using Testosterone Diagnosed by Cardiac MRI
}

\author{
Bhavi Pandya ${ }^{1 *}$, Adarsh Vennepureddy ${ }^{1}$, Neeraj Shah ${ }^{1}$, Deepak Asti ${ }^{2}$, Nikhil Nalluri ${ }^{1}$ and Ruben Kandov ${ }^{2}$ \\ ${ }^{1}$ Department of Internal Medicine, Staten Island University Hospital, NY, USA \\ ${ }^{2}$ Department of Cardiology, Staten Island University Hospital, NY, USA
}

"Corresponding author: Bhavi Pandya, MBBS, Department of Internal medicine, Staten Island University Hospital, 475 Seaview Avenue, Staten Island, NY 10305 , USA, Tel: +1 9099643904; E-mail: dr.bhavipandya@gmail.com

Received date: May 27, 2015, Accepted date: Jul 08, 2015, Published date: Jul 20, 2015

Copyright: (C) 2015 Pandya B, et al. This is an open-access article distributed under the terms of the Creative Commons Attribution License, which permits unrestricted use, distribution, and reproduction in any medium, provided the original author and source are credited.

\section{Case Presentation}

A 39-year-old male with past medical history of hypothyroidism, hyperlipidemia and asthma presented to our emergency room with sub-sternal crushing type of chest pain. The pain was ongoing for the past hour, present at rest, $8 / 10$ in intensity, non-radiating and was not relieved by sublingual nitroglycerine. Two days prior to presentation, the patient had flu like symptoms with low-grade fever, myalgia, sore throat and nasal congestion. He was a non-smoker and denied any illicit drug abuse. The patient had no significant family history. The patient's home medications included inhaled fluticasone-salmeterol, simvastatin, levothyroxine and testosterone injection $200 \mathrm{mg}$ IM once every 2 weeks, which he had been taking for 6 months.

In the emergency room, he was afebrile and his vital signs were stable. Cardiovascular and respiratory examination was normal. His initial EKG showed $1 \mathrm{~mm}$ ST elevations in lead I and aVL with reciprocal ST depressions in lead III and aVF (Figure 1). His initial troponin was 2.08 , which later peaked to 40 , and his initial CKMB value was 26.6 , which later peaked to 77.6 . His chest radiograph was normal.

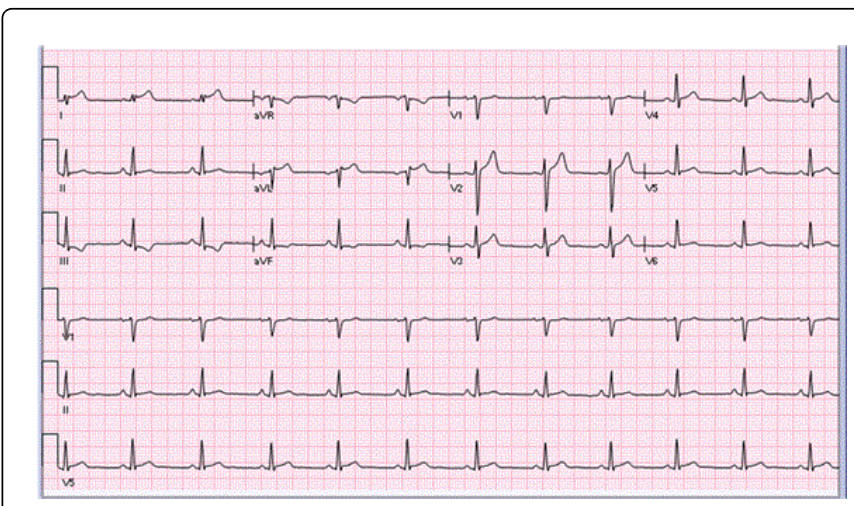

Figure 1: EKG showing ST elevations in lead I and aVL with reciprocal depressions in lead III and aVF.

Patient was initially given aspirin $325 \mathrm{mg}$, clopidogrel $300 \mathrm{mg}$ and was started on IV heparin drip at 1000 units/hour after a bolus of 5000 units. Subsequently, the patient underwent coronary catheterization, which showed normal coronaries with no occlusion. A transthoracic echocardiogram showed $50-55 \%$ ejection fraction with mild hypokinesis of the mid anteroseptal wall.

Since myocarditis was high on the differential list, we decided to do a cardiac MRI. The cardiac MRI showed a large mid myocardial to epicardial post contrast enhancement primarily within the apex, distal lateral and septal walls suggestive of myocarditis. The patient was then started on colchicine and aspirin $81 \mathrm{mg}$. We attributed the myocarditis to be either of viral etiology or secondary to his testosterone usage and the patient was advised to stop testosterone.

The patient was discharged on hospital day 4. At the time of discharge, he was clinically stable and asymptomatic. He refused to stop testosterone and was discharged with close outpatient follow up. His medications on discharge were aspirin $81 \mathrm{mg}$, colchicine $0.6 \mathrm{mg}$, lopressor $12.5 \mathrm{mg}$ bid, simvastatin and synthroid. The patient needed to be on both aspirin and colchicine for 3 months.

Myocarditis is one of the leading causes of chest pain and elevated myocardial enzymes in young patients who have chest pain with no history of coronary artery disease (CAD). In this patient, young age, history of flu like symptoms and use of exogenous testosterone led to high suspicion of myocarditis in the setting of a normal coronary anatomy on cardiac catheterization. Definitive diagnosis of myocarditis can be established by an endomyocardial biopsy; however, it has limited sensitivity and is an invasive procedure. In the past, indium-111 antimyosin antibody was used as diagnostic approach, but it is not used in current practice because of limited availability, radiation exposure and 48-hour delay in obtaining images after injection [1]. We can establish a diagnosis of myocarditis using cardiac MRI, which is non-invasive, safe, highly sensitive and specific, relatively cheap and has no radiation exposure. Cardiac MRI can detect myocardial edema and myocyte damage as well as analyze functional parameters such as left ventricular function, regional wall motion, and flow properties. The contrast enhancement in myocarditis originates primarily from the epicardium sparing the sub endocardial layer whereas in myocardial infarction cardiac MRI typically shows sub-endocardial enhancement [2]. The MRI in our patient showed the characteristic patchy epicardial/mid myocardial post contrast enhancement of the apex, distal lateral and septal walls sparing the sub endocardial layer suggestive of myocarditis $[3,4]$. In acute myocarditis, T2 weighted cardiac MRI images show myocardial edema usually observed in the lateral wall, localizing in the sub epicardial layer and following a non-coronary distribution (Figure 2), whereas in myocardial infarction, myocardial edema distributes in the region of coronary artery supply. T2 weighted cardiac MRI visualizes myocardial edema that corresponds to ischemia, active inflammation, vasculitis or recently performed intervention in the myocardium and provides information complementary to delayed enhancement MRI [3] (Figures 2-4). 
Citation: Bhavi Pandya, Adarsh Vennepureddy, Neeraj Shah, Deepak Asti, Nikhil Nalluri and Ruben Kandov (2015) Acute Myocarditis in a Patient Using Testosterone Diagnosed by Cardiac MRI. J Steroids Horm Sci 6: 1000157.doi: 10.4172/2157-7536.1000157

Page 2 of 3

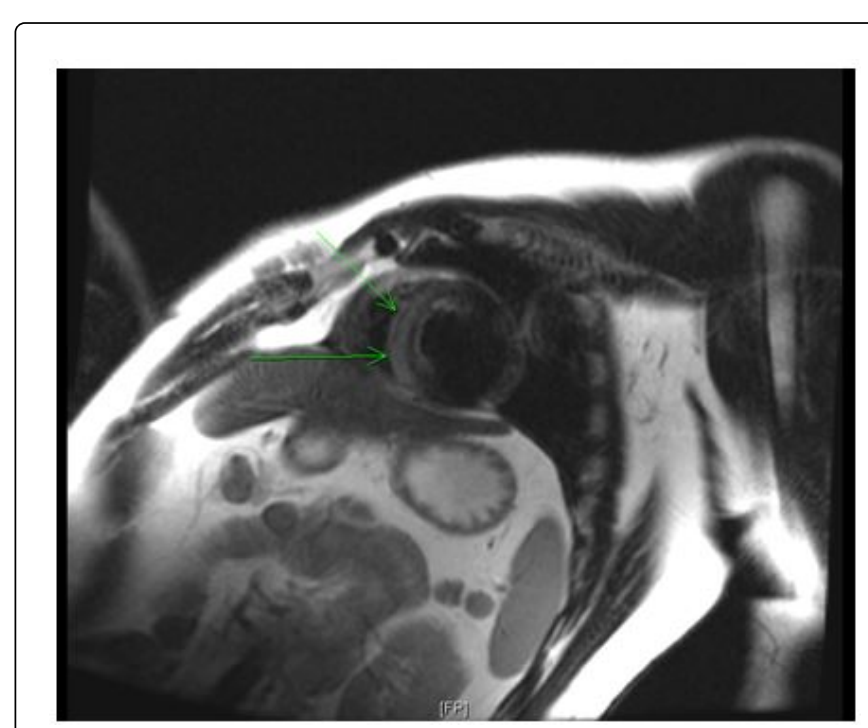

Figure 2: Double inversion recovery T2 weighted short axis image showing acute edema in interventricular septum at mid ventricular level.

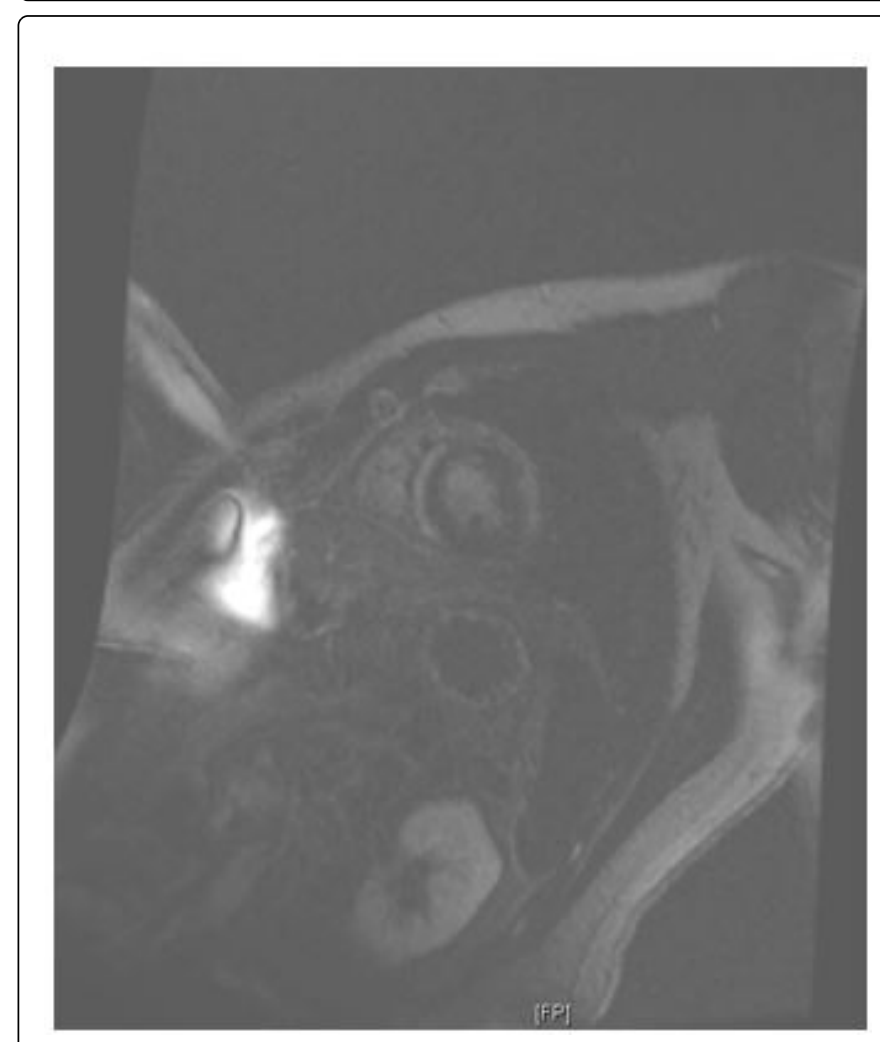

Figure 3: Delayed post contrast enhancement short axis images showing mid myocardial to epicardial enhancement which is circumferential in apex and extending into distal, lateral wall and septum into mid ventricular level.

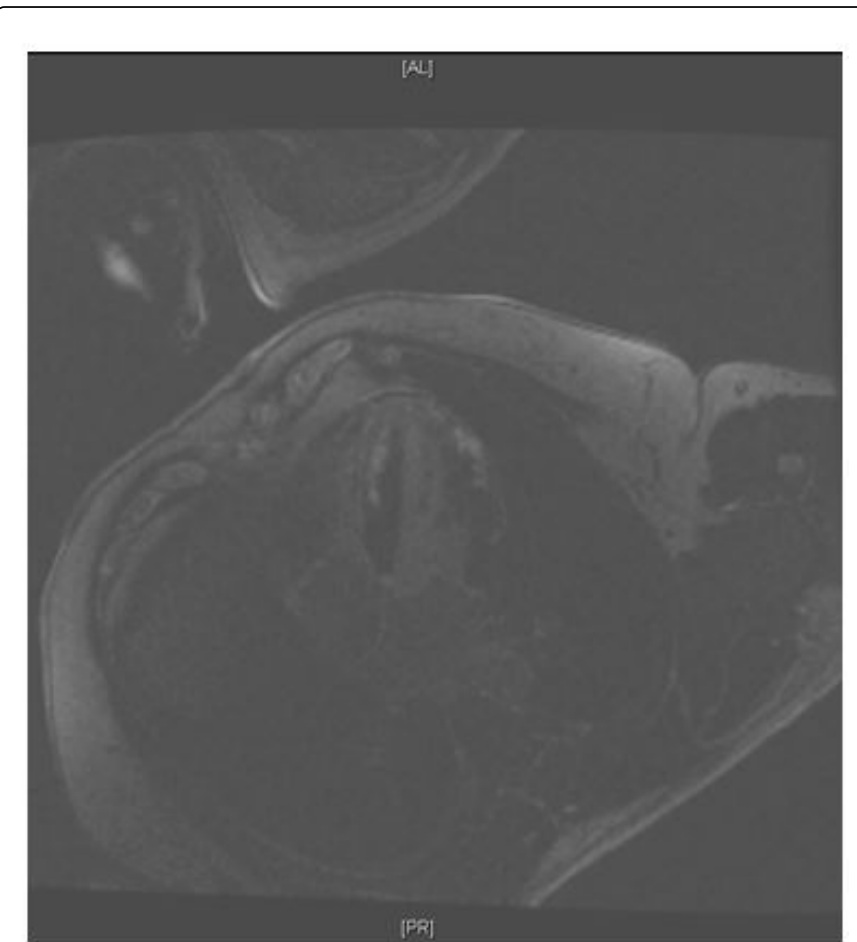

Figure 4: Delayed post contrast enhancement 4 chamber view.

Moreover, we observe that myocarditis occurred in this patient approximately 5 months after he started using exogenous testosterone replacement therapy. Testosterone has often been implication in being responsible for higher prevalence of myocarditis in males compared to females. Testosterone is associated with selective induction of CD4+ TH1 (IFN- $\gamma$ positive) response, which is pro-inflammatory, whereas estradiol is associated with CD4+ TH2 (IL 4) response, which is antiinflammatory. Testosterone increases the expression of proinflammatory cytokines in the heart like TNF alpha, IL 1 beta, IL 6 and IFN- $\gamma$ and activates apoptotic genes like p38, MAP kinase and caspase 1,3 and 11, which can lead to myocardial inflammation and fibrosis [4]. Testosterone also increases the susceptibility of myocardium to coxsackievirus $\mathrm{B}$ by increasing the virus receptor expression on endothelial cells and myocytes [5] as well as toll like receptor 4 (TLR4) on monocytes and macrophages. Testosterone inhibits the antiinflammatory populations in the heart including $\mathrm{T}$ cell immunoglobulin mucin (Tim 3) on macrophages, Tim 3+ CD4+ T cells, $\mathrm{TH} 2$ and regulator $\mathrm{T}$ cells resulting in more severe acute cardiac inflammation following coxsackie B virus infection [6]. Even though viral infection is a more likely cause of myocarditis in this patient, the use of testosterone may have played a significant role, especially in terms of producing a more severe form of myocarditis with ST elevations on EKG and a peak troponin level of 40, thus indicating significant myocardial involvement and damage.

In summary, in a young patient on exogenous testosterone replacement with no significant $\mathrm{CAD}$ risk factors, typical chest pain and elevated troponins are suggestive of myocarditis rather than myocardial ischemia even in the face of significant EKG changes. In facilities equipped with inpatient cardiac MRI, it may be safer to initially perform a cardiac MRI instead of invasive coronary imaging in these patients. Cardiac MRI is useful in differentiating myocarditis 
Citation: Bhavi Pandya, Adarsh Vennepureddy, Neeraj Shah, Deepak Asti, Nikhil Nalluri and Ruben Kandov (2015) Acute Myocarditis in a Patient Using Testosterone Diagnosed by Cardiac MRI. J Steroids Horm Sci 6: 1000157.doi:10.4172/2157-7536.1000157

Page 3 of 3

from myocardial ischemia, infarction, and coronary artery spasm. The patients with myocarditis should be advised to restrict the use of exogenous testosterone as it may increase the incidence and severity of future episodes of myocarditis.

\section{References}

1. Jeserich, M (2009) Non-invasive imaging in the diagnosis of acute viral myocarditis. Clin Res Cardiol 98: 753-763.

2. Testani, JM (2006) Focal myocarditis mimicking acute ST-elevation myocardial infarction: diagnosis using cardiac magnetic resonance imaging. Tex Heart Inst J 33: 256-259.
3. Amano Y (2012) T2-weighted cardiac magnetic resonance imaging of edema in myocardial diseases. Scientific World Journal 94069.

4. Wang, M (2005) Role of endogenous testosterone in myocardial proinflammatory and proapoptotic signaling after acute ischemiareperfusion. Am J Physiol Heart Circ Physiol 288: H221-226.

5. Lyden, DC (1987) Coxsackievirus B-3-induced myocarditis. Effect of sex steroids on viremia and infectivity of cardiocytes. Am J Pathol 126: 432-438.

6. Frisancho-Kiss S (2009) Gonadectomy of male BALB/c mice increases Tim-3(+) alternatively activated M2 macrophages, Tim-3(+) T cells, Th2 cells and Treg in the heart during acute coxsackievirus-induced myocarditis. Brain Behav Immun 23: 649-657. 